\title{
HISTORIA
}

\section{LA FAMILIA, LA ENFERMEDAD, EL DOLOR Y LA MUERTE EN LA OBRA PICTÓRICA DE EDVARD MUNCH}

Morillo Cadierno R.M.; González Moreno M.J.; Capado Rodríguez M.M. Departamento de Enfermería. Escuela Universitaria de Ciencias de la Salud de la Universidad de Sevilla

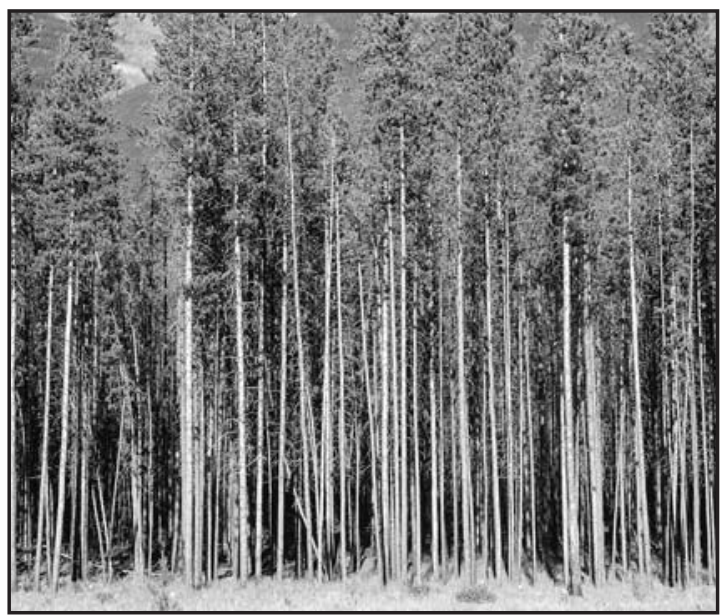

FAMILY, DISEASE, PAIN AND DEATH IN THE PICTORIAL WORK OF EDVARD MUNCH

\section{SUMMARY}

INTRODUCTION: Disease, pain and death are very significant events in life thus it is natural to see them reflected in pictorial works of art. Considering Edvard Munch had a severe experience of illness and death in his own family when child, he felt the need to deepen and analyzing his life and work to discover the intrinsic meaning of disease and death to illustrate some of his canvases.

METHODOLOGY: For an adequate evaluation and analysis Munch's life, work and historical context was studied. The visual support for this communication has been extracted from diverse pictorial works directly concerned with terms as family, pain, disease and death.
CONCLUSIONS: Painting constitutes a vehicle to express and show disease, death and to deepen in their implicit ones. Munch like a main character of a Greek tragedy painted his environment, he showed what he saw: his sister's illness, his family's pain, his mother's death and his patient awaiting of his own death. Much's work of art is like a dramatic, raw, real and unique a universe created by a tormented and solitary genius who suffered like no other human being the experience of pain, disease and death in his own family.

Key-words: Family, disease, death

\section{A FAMÍlIA, A DOENÇA, A DOR E A MOTE NA OBRA PICTÓRICA DE EDVARD MUNCH}

\section{RESUMO}

INTRODUÇÃO: A enfermidade, a dor e a morte são acontecimentos muito significativos, sendo algo natural que apareçam nas obras pictóricas. Considerando o fato de que, desde sua infância Edvard Munch sofreu a experiência da enfermidade e a morte na sua própria família, surgiu a necessidade de realizar uma análise de sua vida e obra para desvelar o significado intrínseco desses fenômenos que ilustram alguns de suas pinturas.

METODOLOGIA: Para a análise e valorização adequadas do objeto, foram analisadas a vida e obra de Munch, bem como sua contextualização histórica. O recurso visual dessa comunicação está 
constituído por diversas obras pictóricas que se revelam relavantes para os vocábulos: família, dor, enfermidade e morte.

CONCLUSÕES: A pintura constitui um veículo para identificar a doença, a morte e possibilitar a discussão sobre suas implicações. Munch, como protagonista de uma tragédia grega, pintou o que observou: a enfermidade de sua irmã, a dor de sua família, a morte de sua mãe e a espera paciente de sua própria morte. A obra pictórica de Munch exposta aparece como um universo cru, dilacerante, real e irrepetível, criada por um gênio atormentado e solitário, que padeceu como poucos a experiência da dor, a enfermidade e a morte em sua própria família.

Palabras-chave: Família, Enfermidade, Morte.

\section{RESUMEN}

INTRODUCCIÓN: La enfermedad, el dolor y la muerte son acontecimientos muy significativos que resulta natural que aparezcan en las obras pictóricas. Teniendo en cuenta, que desde su niñez Edvard Munch sufrió la experiencia de la enfermedad y la muerte en su propia familia, surgió la necesidad de realizar un análisis de su vida y obra para descubrir el significado intrínseco de la enfermedad y la muerte que ilustran algunos de sus lienzos.

METODOLOGÍA: Para el análisis y valoración adecuados se estudió la vida y obra de Munch; así como su contextualización histórica. El soporte visual de esta comunicación lo constituyen diversas obras pictóricas que atañen directamente a los términos: familia, dolor, enfermedad y muerte.

CONCLUSIONES: La pintura constituye un vehículo para reseñar la enfermedad, la muerte y profundizar en sus implícitos. Munch, como el protagonista de una tragedia griega, pintó lo que vio: la enfermedad de su hermana, el dolor de su familia, la muerte de su madre y la espera paciente de su propia muerte. La obra pictórica de Munch expuesta aparece como un universo crudo, lacerante, real e irrepetible creada por un genio atormen- tado y solitario que padeció como pocos la experiencia del dolor, la enfermedad y la muerte en su propia familia.

Palabras Clave: Familia, enfermedad, muerte

\section{INTRODUCCIÓN}

La enfermedad, el dolor y la muerte son acontecimientos muy significativos que resulta natural que aparezcan en las obras pictóricas. Teniendo en cuenta, que desde su niñez Edvard Munch sufrió la experiencia de la enfermedad y la muerte en su propia familia, surgió la necesidad de realizar un análisis de su vida y obra para descubrir el significado intrínseco de la enfermedad y la muerte que ilustran algunos de sus lienzos.

El Arte de Munch, conceptualmente, es, ante todo, introspectivo, existencialista. Su pintura es el vehículo de su angustia y ésta a su vez es la fuente de aquélla.

"Sin el miedo y la enfermedad mi vida sería como un bote sin remos"

Con estas palabras resumía Edvard Munch el sentido de su pintura. Al igual que otros artistas, la enfermedad, el dolor y la muerte; marcaron de tal forma su vida que el pintor nunca pudo deshacerse del hombre que lo envolvía. "Yo no pinto lo que veo, sino lo que vi" decía Munch. Y lo que vio fue plasmado en su pintura como una percepción momentánea de una escena de la existencia humana.

Quizás estemos acostumbrados al sufrimiento, al dolor en soledad. Podemos realizar el esfuerzo, ahora, durante apenas un instante, lo extremadamente difícil que sería expresarnos desde cualquier técnica artística obviando el sufrimiento, la melancolía y la soledad del dolor. Como un acto agónico, como el protagonista de una tragedia griega, Munch pintó lo que vio: un grito callado desde la cama, un marco inamovible de blancos radiantes, un soporte para la composición del lienzo y de su vida. Munch, el pintor de almohadas.

Desde su niñez, la muerte y la enfermedad ocuparon un lugar central en su vida. Con cinco años ve cómo su madre muere de tuberculosis, enfermedad que se llevará también a su querida hermana Sophie a la edad de 15 años. En noviembre de 1889 fallece también su padre. 
Él mismo dejó constancia de su ánimo sombrío: "Enfermedad, muerte y locura fueron los ángeles negros que velaron mi cuna y desde entonces me han perseguido durante toda mi vida". Se ha debatido mucho sobre la naturaleza de la enfermedad mental de Munch. Se ha hablado de temperamento melancólico, de síndrome ansiosodepresivo, de fobia social, de agorafobia, de neurosis y hasta de esquizofrenia. Sin embargo parece que no padeció esquizofrenia. Quizá sólo tuvo un trastorno de la personalidad, caracterizado por la introversión, así como un excesivo consumo de alcohol. Sea como fuere, en la última etapa de su vida pareció reconciliarse con sus espectros.

\section{METODOLOGÍA}

Para el análisis y valoración adecuados se estudió la vida y obra de Munch; así como su contextualización histórica. El soporte visual de esta comunicación lo constituyen diversas obras pictóricas que atañen directamente a los términos: familia, dolor, enfermedad y muerte.

\section{VIDA Y OBRA DE EDVARD MUNCH}

Munch nació en Löten, Noruega, en 1863, era hijo de un médico militar y en su familia había una gran tradición de artistas e intelectuales. Pero no fue este pasado ilustre lo que marcó su vida sino la muerte. Cuando apenas tenía cinco años falleció su madre. Esto llevó a que su padre, un hombre profundamente religioso, tuviera crisis místicas. A los pocos años falleció su hermana más querida, su otra hermana cayó en depresiones profundas (llamadas entonces "melancolía") y sólo su hermano varón llegó a edad adulta, pero a los pocos días de casarse también falleció. Munch sentía cómo la sombra de la muerte dominaba toda su vida.

A los diecisiete años decidió que quería ser pintor y partió hacia Cristiania, la capital de Noruega. Estudió en la Escuela Real de Dibujo y se convirtió en discípulo de Christian Krogh, el más importante pintor naturalista noruego quien descubrió enseguida las cualidades artísticas de su alumno.

En 1885 partió en viaje de estudios a París donde descubrió a los impresionistas que revolucionaban el arte francés. Munch se dio cuenta que el naturalismo ya no tenía sentido y que debía pintar no lo que veía sino lo que sentía. Volvió a
Noruega con estas ideas y se puso a trabajar en una obra que reflejara sus nuevas ideas en el arte.

El resultado fue una obra maestra y monumental: "Niña enferma", una obra desgarradora donde a partir de trazos gruesos y colores contrastantes, Munch conseguía transmitir el dolor por su hermana muerta. La crítica noruega, recibió despectivamente a esta obra que parecía despreciar los principios básicos del arte naturalista. Debido a esa respuesta, Munch pintó algunas telas más tradicionales.

En 1889 su vida iba a cambiar rotundamente al conocer a Hans Jæger, el líder artístico y político de los anarquistas noruegos. Jæger había estado en la cárcel debido a sus principios políticos y también sexuales, ya que fue uno de los impulsores del amor libre en Noruega. Munch se fascinó con este gran anarquista y compartía su ideología rebelde.

Ese mismo año viajó nuevamente a París en el que comenzaba la etapa post-impresionista marcada también por un profundo desprecio por el arte naturalista.

También fue en París donde se enteró de la muerte del único ser de su familia que le quedaba: su padre. Una vez más Munch sufrió una depresión marcada por la sensación de estar solo en el mundo. Sin embargo, siguió trabajando en sus pinturas que aún mantenían una gran influencia de los impresionistas franceses.

Hacia 1891, Munch decidió ir hacia una búsqueda todavía más arriesgada y personal. En esa década de los 90 iba a crear sus cuadros más logrados y célebres "El grito" cuyos primeros bosquejos son de 1891, aunque su versión más famosa es de 1893. De "El grito" existen alrededor de cincuenta versiones.

Con su nuevo estilo tenía como tarea "estudiar el alma”. Con este bagaje de obras llegaría Munch en 1893 a Berlín para una muestra individual en una prestigiosa galería.

El rechazo de los primeros años se convirtió de manera casi automática en admiración. Munch pasó a ser un artista respetado y admirado, aunque Noruega tardó algunos años más en reconocer la grandiosa obra de su hijo pródigo.

Pero el creciente éxito como artista no iba acompañado con una vida de felicidad. Sus relaciones con las mujeres siempre fueron siempre conflictivas. 
Al igual que los anarquistas que frecuentaba, Munch estaba a favor del amor libre. Durante mucho tiempo estuvo enamorado de una amiga de su infancia, pero también ella, su amor loco de juventud, murió joven.

En 1898, Munch tenía una relación tormentosa con otra chica; actos de amor y de desamor se sucedían entre los dos, que finalmente acabaron con la relación sentimental. Desde entonces, Munch prefirió la soledad.

El artista se refugió en el alcohol y los fantasmas de muerte y dolor se multiplicaron. En 1908 tuvo que ser internado en un instituto psiquiátrico en Copenhague durante varios meses donde fue sometido a descargas eléctricas para lograr su curación. Cuando salió de la clínica decidió volver a Noruega. Estaba mucho más tranquilo y pero los autorretratos de esa época lo muestran agotado. $\mathrm{Su}$ pintura había perdido parte de ese trazo nervioso que lo había identificado. Igualmente, no perdió su genio tal como lo testimonian los murales del aula magna de la Universidad de Oslo y en las pinturas de los paisajes noruegos.

Munch acumulaba las telas en su casa de Oslo porque no le gustaba venderlas ya que consideraba que la obra tomaba su real sentido en el conjunto y no individualmente. Por eso, reproducía por distintos medios sus pinturas, en xilografía y en litografía, entre otros procedimientos en los que se destacó.

"Enfermedad, locura y muerte fueron los ángeles negros que velaron mi cuna al nacer" escribió una vez. Munch murió a los 81 años, en una Oslo invadida por los nazis. Murió en soledad, rodeado por cientos de sus cuadros.

Tras un breve repaso de la vida de Munch se comentarán las obras elegidas para esta comunicación:

\section{La niña enferma}

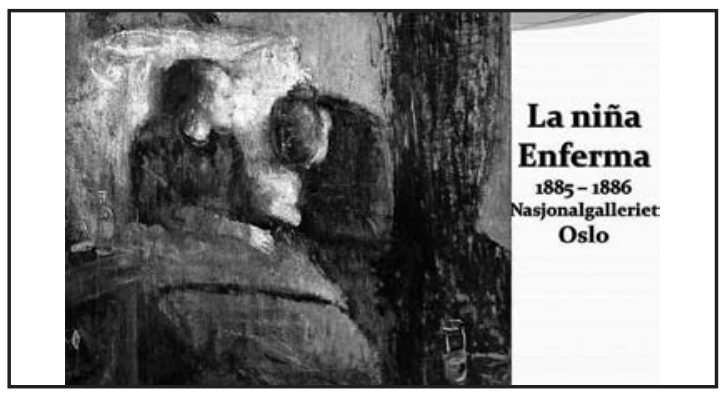

Si alguien nos habla de Munch, inmediatamente visualizamos El grito, pero a mi juicio, pintó una obra anterior a ésta que produce en mi espíritu un sentimiento que me perturba por su realismo desgarrador: crudo realismo desde el impresionismo. Su primera obra maestra: La niña enferma. Os animo a que la contempléis conmigo:

La niña enferma (por supuesto evocando la enfermedad de la hermana de Munch, Sophie) está sentada en la cama, enderezada por una gran almohada de plumas que la sujeta. Su cara vuelta hacia el lado donde una mujer sin rostro parece estar sentada o arrodillada. En la parte izquierda, una cómoda en la que reposa una botella nos hace pensar en un estrecho espacio; un trozo de pared que parece confundirse con la almohada; en la parte derecha, una superficie verdosa, quizás una cortina cerrada y en primer plano, una mesita con un vaso de agua. Ese es el mundo del dolor: cerrado. ¡No se puede respirar! ¡No hay esperanza!. Las figuras humanas y los objetos están encerrados como peces en un acuario.

Podemos sentir el dolor incontenible de una madre sin rostro inclinada ante lo irremediable, ante el respeto que nos produce la muerte y, entregada (las manos de ambas, madre e hija, están entrelazadas) a una hija que la contempla con resignación y paciencia. El dolor del que produce dolor. Fijaros en los ojos de la niña. Su mirada tranquila y sosegada. La mirada de la aceptación, del peor dolor del otro: querer morirse.

A pesar de lo que pueda sugerirnos este lienzo, fue fuertemente criticado cuando en 1886 fue exhibido por primera vez durante la Exposición de Otoño de Cristianía. Ni el tema ni la composición eran motivo de escándalo. La forma que fue ejecutado con sólo esbozos escarbados en el lienzo y los surcos que parecían arañar la tela poniendo de manifiesto una intensa agitación nerviosa pueden explicar el desasosiego de los críticos. Ahora, os invito a que lo contempléis con los ojos de Munch. Los múltiples retoques, los rasguños que penetran en todas las capas de pintura, las sombras capaces de modelar los cuerpos y, la luz. Una luz que no incide desde fuera sino del interior de las figuras, de la gran almohada y del rostro trasparente de la niña enferma. Nada en Munch queda inacabado. Una obra de arte está completa cuando el artista ha 
dicho todo lo que tenía en su corazón. Y en "La niña enferma" no quedó nada por decir.

En La niña enferma, tema sobre el que Munch volverá una y otra vez, la emoción no emana únicamente de la escena, sino también de la técnica, pues los trazos toscos e incompletos parecen revelar unas manos temblorosas pero a la vez incisivas, que hubieran pretendido arañar, más que pincelar, la tela.

\section{La primavera. 1889}

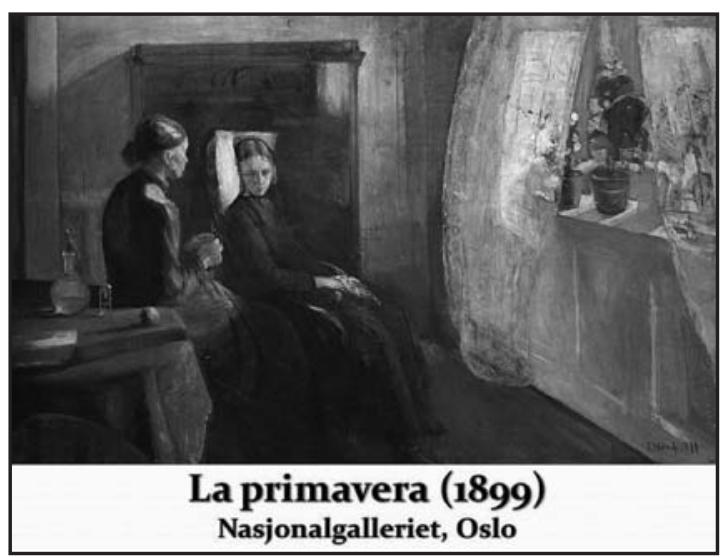

El año 1889 comienza con una enfermedad grave de Munch. Durante su convalecencia, Munch pinta La primavera y despliega todo el espectro de la técnica naturalista aplicada a la reproducción de un interior.

En el centro del cuadro se encuentra sentadauna vez más- la niña enferma enmarcada por un cojín blanco y por un enorme armario marrón al fondo. La extraordinaria palidez de su rostro contrasta con el color saludable de la figura que aparece de perfil, una señora algo mayor sentada al lado de la enferma, tal vez la madre. La jarra de cristal, así como los frascos de medicamentos sobre la mesa oscura, aparte de acompañar a la enferma, forman el polo opuesto a las macetas alineadas sobre el alféizar de la ventana por donde entra la luz del día. Las cortinas ligeramente abombadas por una corriente de aire aluden claramente a la fuerza curativa del aire fresco que penetra en la oscuridad de la alcoba. Parece como si los objetos presentes en la escena tuvieran la capacidad de hablar.

\section{Junto al lecho de muerte. 1895}

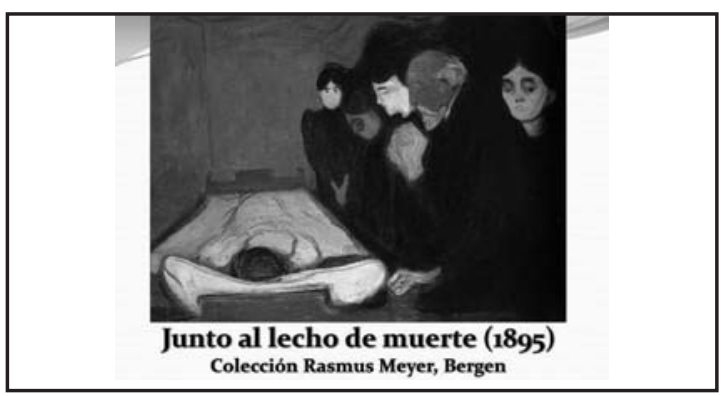

En esta obra ya no es la enfermedad el tema del cuadro, el tema es la familia y la muerte. Los protagonistas de la escena son los miembros de la familia de Munch cuyos rostros y manos surgen de una zona oscura. Gracias al tratamiento del lecho, casi plano y escorzado, el espectador se convierte en testigo presente, en visita del enfermo. Se puede observar claramente que la lividez de las sábanas contrasta con el marrón rojizo de las paredes. Por la derecha se aproxima la sombra de la muerte, destacándose de ella los rostros, pintados con trazos de los allegados. Su identidad permanece indeterminada por la escasez de rasgos. Sin embargo, podemos reconocer al padre de Munch que ora fervorosamente. El mismo Munch podría estar a su lado. Pero no es posible saber si la mujer del primer plano es la tía Karen, quién se hizo cargo de la familia tras la muerte de la madre de Munch y quién apoyó a éste cuando tomó la decisión de ser pintor, así como tampoco se puede decir con precisión, si las figuras femeninas del fondo son sus hermanas Inger y Laura.

\section{Muerte en la habitación. 1895}

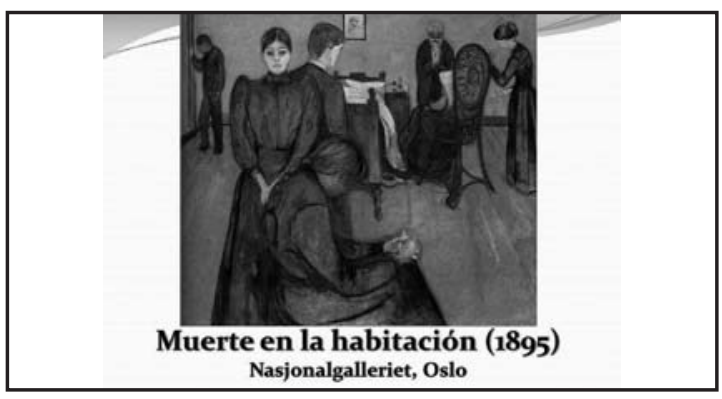

Este cuadro fue pintado en 1895, parece que se puede palpar la desesperante estrechez de la habita- 
ción aislada del mundo exterior. El alto respaldo del sillón de mimbre oculta al enfermo que acaba de morir. Las hermanas de Munch Inger y Laura (en primer plano, de pie y sentada respectivamente) son fáciles de reconocer. $\mathrm{Al}$ fondo, el pintor vuelve la espalda a la escena. En torno al sillón se encuentran el padre y la tía Karen. La otra figura masculina podría ser Andreas, el hermano de Munch.

La suma de la meditación de Munch sobre la muerte está contenida en este cuadro.

\section{Madre muerta con niña (1897-1899)}

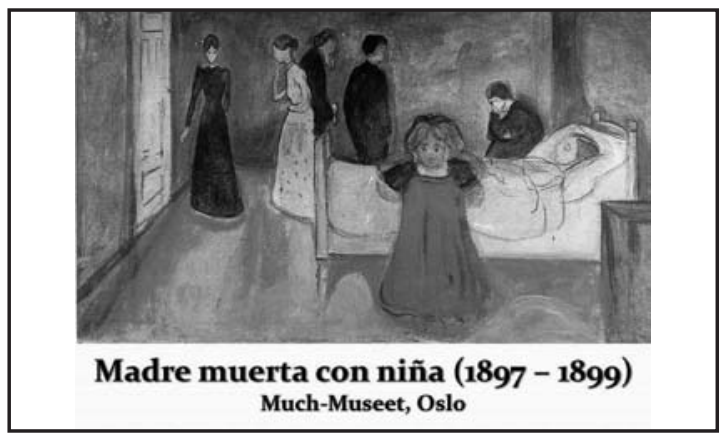

En este cuadro, el tema es -una vez más- la muerte. Munch rememora de nuevo la temprana muerte de su madre en 1868 .

Detrás de la cama colocada horizontalmente en el cuadro, se encuentran cuatro personas adultas de la familia que expresan mediante gestos nerviosos y reservados, y en su ir y venir cabizbajos, su impotencia frente a la muerte. Sophie, en cambio, se halla en el primer plano vuelta hacia el espectador: se ha tapado los oídos con las manos como si quisiera ponerse a salvo del dolor que le produce el silencioso grito de la muerte.

\section{Madre muerta con niña (1899-1900)}

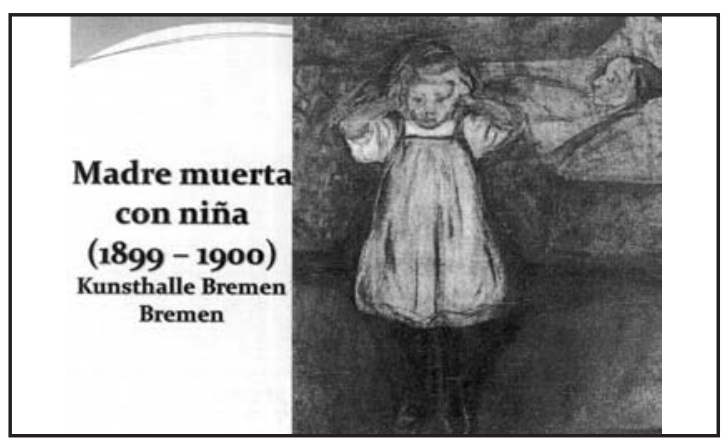

En la versión posterior del cuadro Madre muerta con niña de 1899-1900, que está en la Galería de Bremen, los miembros de la familia han desaparecido; lo que ha quedado es tan sólo un retrato de su hermana Sophie. En una vertical monumental, la niña cuya madre yace muerta en la horizontal nos hace frente. La pintura muestra en el fondo, la cama en donde reposa el cadáver de una mujer, y en el primer plano, una niña de mirada ausente que se tapa los oídos con fuerza. La niña, ahí presente, lo es todo: el vacío, la desesperanza, la angustia, la soledad y el terror, ciertos aspectos que se repiten a menudo en la obra del pintor noruego.

Así como el genio del Renacimiento Leonardo Da Vinci diseccionaba cuerpos con la intención de conocer su estructura y funcionamiento, Munch pretendía diseccionar las almas.

$\mathrm{Y}$ volviendo al cuadro de Madre muerta con niña, siempre he sentido un cariño especial hacia él. Tal vez por la niña, que intenta tapar sus oídos de todo el ruido, de todo el llanto, de todo el caos exterior y crea una burbuja casi invisible a su alrededor. Tal vez por el aislamiento al que los adultos confinan a la niña, concentrándose en su propio duelo, en su propio llanto, sea fingido o sea real. O tal vez por el vacío en la mirada de la niña, de los dolientes, de todos los personajes que Munch presenta en su obra. Tal vez es el vacío con quién siento esa empatía...

\section{Autorretrato entre el reloj y la cama (1940- 1942)}

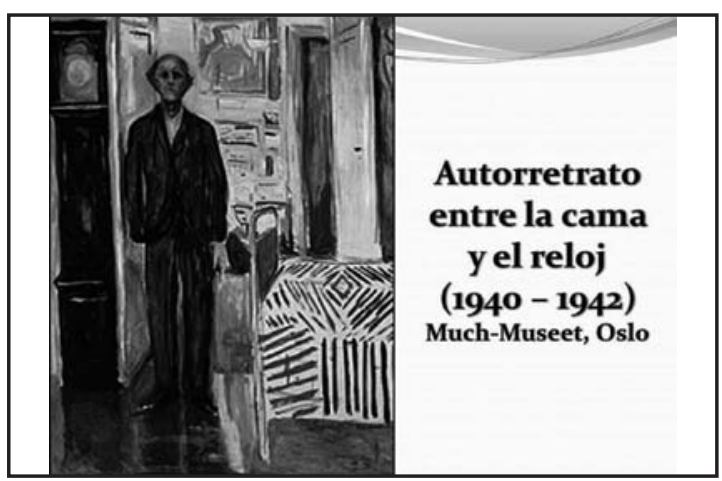

Por último, uno de sus últimos cuadros, Autorretrato entre el reloj y la cama, de 1940. El 
reloj no tiene más agujas, la cama, austera como la cama de un hospital, es dónde Edvard Munch murió.

Enflaquecido, caquéctico, es un anciano, está ya esperando la muerte, sus ojos y su boca ya están cerrados.

Sus cuadros están en la pared, testimonio de su última vida.

\section{Autorretrato junto a la ventana (1942)}

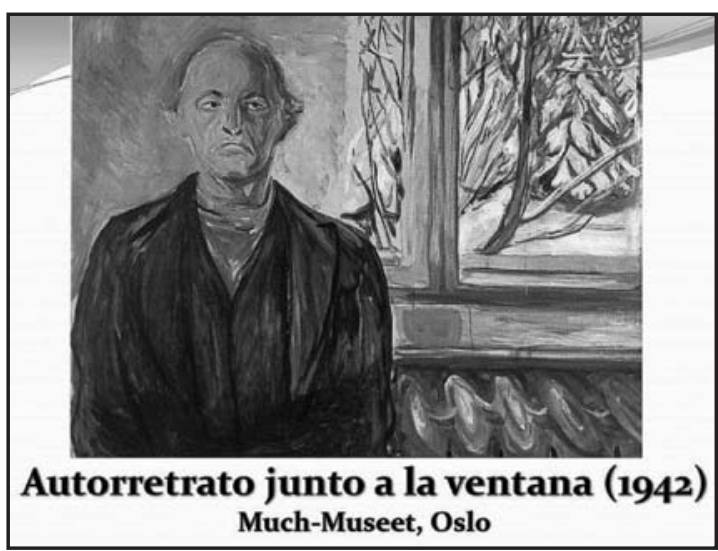

Este último cuadro de formato alargado es la viva imagen de la oposición entre la vida y la muerte. El rojo intenso de la cara y la pared, así como el verdiazul de la ventana forman un bastión contra la muerte representada por un paisaje helado que penetra por la ventana.

La estructuración vertical del formato del cuadro acentúa la polaridad entre el yacer y el estar de pie.
La vida humana es una provisional victoria sobre la fuerza de la gravedad, sobre la materia. El pintor se incorpora por última vez para acostarse a morir. Su vida es el símbolo de esa victoria. Como tal símbolo, el cuadro reconcilia la vida con la muerte, la línea vertical con la horizontal, el movimiento con el reposo.

CONCLUSIONES: La pintura constituye un vehículo para reseñar la enfermedad, la muerte y profundizar en sus implícitos. Munch, como el protagonista de una tragedia griega, pintó lo que vio: la enfermedad de su hermana, el dolor de su familia, la muerte de su madre y la espera paciente de su propia muerte. La obra pictórica de Munch expuesta aparece como un universo crudo, lacerante, real e irrepetible creada por un genio atormentado y solitario que padeció como pocos la experiencia del dolor, la enfermedad y la muerte en su propia familia.

Munch murió a los 81 años y efectivamente pintó lo que vio.

\section{BIBLIOGRAFÍA}

- BISHOFF, U, Edvard Munch. 1863-1944. (2000) Cuadros sobre la vida y la muerte, Taschen, Colonia.

- BOE, A. (1989) Edvard Munch, versión fançaise de Robert Marrast, Polígrafa, Barcelona.

- CHIPP, H. B. (1995) Teorías del arte contemporáneo. Fuentes artísticas y opiniones críticas. Traducción de Julio Rodríguez Puértolas, Akal, Madrid.

- HODIN, J. P. (1994) Edvard Munch, Ediciones Destino, Barcelona.

- ROSENBLUM, R. (1993) La pintura moderna y la tradición del Romanticismo nórdico. De Friedrich a Rothko, Alianza, Madrid. 